\title{
Structure, innervation, mechanical properties and reflex activation of a striated sphincter in the vestibule of the cat vagina
}

\author{
Roberto Lagunes-Córdoba ${ }^{1}$, Víctor Tsutsumi ${ }^{2}$ and E J Muñoz-Martínez ${ }^{1}$ \\ ${ }^{1}$ Departamento de Fisiología, Biofísica y Neurociencias and ${ }^{2}$ Departamento de Patología Experimental, Centro de \\ Investigación y de Estudios Avanzados CINVESTAV del IPN, Apartado Postal 14-740, 07360 México D. F., México
}

Correspondence should be addressed to E J Muñoz-Martínez; Email: jmunoz@fisio.cinvestav.mx

\begin{abstract}
Vaginal constriction might be important for reproduction in mammals, but existing information is both limited and controversial. This paper shows the structure, mechanical properties, innervation and reflex response of a striated sphincter in the vestibule of the cat vagina. A Foley catheter coupled to a pressure transducer detected in the lumen of the vestibule a pressure wave that was induced by stimulation of the external branch of the motor pudendal nerve. The peak pressure of the wave induced by bilateral stimulation $(30.6 \mathrm{~cm}$ $\mathrm{H}_{2} \mathrm{O}$ ) was about double of the peak pressure wave induced unilaterally. The tetanus/twitch amplitude ratio was 4.5 . The sphincter that produces the increase in vaginal pressure fatigues slowly. Digital, point-to-point summation of unilateral waves was greater than the wave induced bilaterally. Summation of the pressure wave induced by the separate stimulation of the terminal motor branches was also greater than the wave induced by the entire motor nerve. This might reflect multiple innervation of muscle fibres. Single, controlled probing of the vaginal vestibule induced a reflex discharge in the motor nerve. Repetitive probing $(10 \mathrm{~Hz})$ induced a motor nerve post-discharge lasting $>1 \mathrm{~min}$. The vaginal sphincter is two-half rings of striated muscle fibres in the wall of the vaginal vestibule; the fibres end freely in the dorsal and ventral midlines. Penetration of the vestibule by the penis might trigger sustained contraction of the vaginal sphincter. Reproduction (2009) $137371-377$
\end{abstract}

\section{Introduction}

Constriction of the distal vagina (the vestibule) occurs in mammals, including humans. This might help to hold the penis during mating (Crouch \& Lackey 1969, Martin et al. 1974, Shafik 1993) and to support the foetus' head during parturition (Shafik 1993). Striated sphincters surrounding the vaginal vestibule were described in fixed post-mortem cats (Crouch \& Lackey 1969, Martin et al. 1974). The two descriptions in the cat show little coincidence. In rats and rabbits, no sphincters but skeletal muscles with one bone insertion apparently produced vaginal constriction (Pacheco et al. 1989, Cruz et al. 2002; see also Martínez-Gómez et al. 1997). In women, vaginal constriction was attributed to smooth muscle (Shafik et al. 2004), although striated muscles cannot be excluded (see Oelrich 1983). The information on the nerves involved in vaginal constriction is also scarce and inconsistent. In the rabbit, stimulation of motor pudendal nerve (MPN) branches induces vaginal constriction (Cruz et al. 2002). In the rat, vaginal constriction was induced by the stimulation of the pelvic nerve (Pacheco et al. 1989).

The present paper shows the innervation, the mechanics and the motor response to vestibule penetration as well as the structure of a sphincter in the wall of the vaginal vestibule. Concerning the innervation, it is a dogma that each fibre in striated muscles receives a single endplate, but several facts show that some fibres might receive more than one endplate from different axons (Jarcho et al. 1952, Rossi 1990, Zenker et al. 1990, Duxson \& Sheard 1995, Happak et al. 1997, Perie et al. 1997, Lateva et al. 2002). Evidence of multiple innervation of vestibular constrictor sphincter (VCS) muscle fibres by both ipsilateral and contralateral MPN axons is shown here.

\section{Results}

\section{Constriction of the vaginal vestibule}

Single shock to the peripheral stump of e-MPN induced a wave-like $(\mathrm{Pv})$ increase in pressure in the vestibular lumen (Fig. 1A). Shock 1.3-1.5 times the threshold intensity ( $\cong 3 \mathrm{~V}, 50 \mu \mathrm{s})$ induced maximal Pv. L, R and LR refers in the text and figures to the left, the right or both e-MPNs or to the Pv induced by these nerves. We will call VCS the muscular apparatus producing Pv. The average peak of Pv-LR (30. $6 \pm 9.6 \mathrm{~cm}$ of water; $N=14$ ) was about twice the peak of unilateral Pv (Fig. 1A and B). Thus, VCS is functionally composed of halves. Average $\mathrm{Pv}$ peak varied among cats; the difference was not 

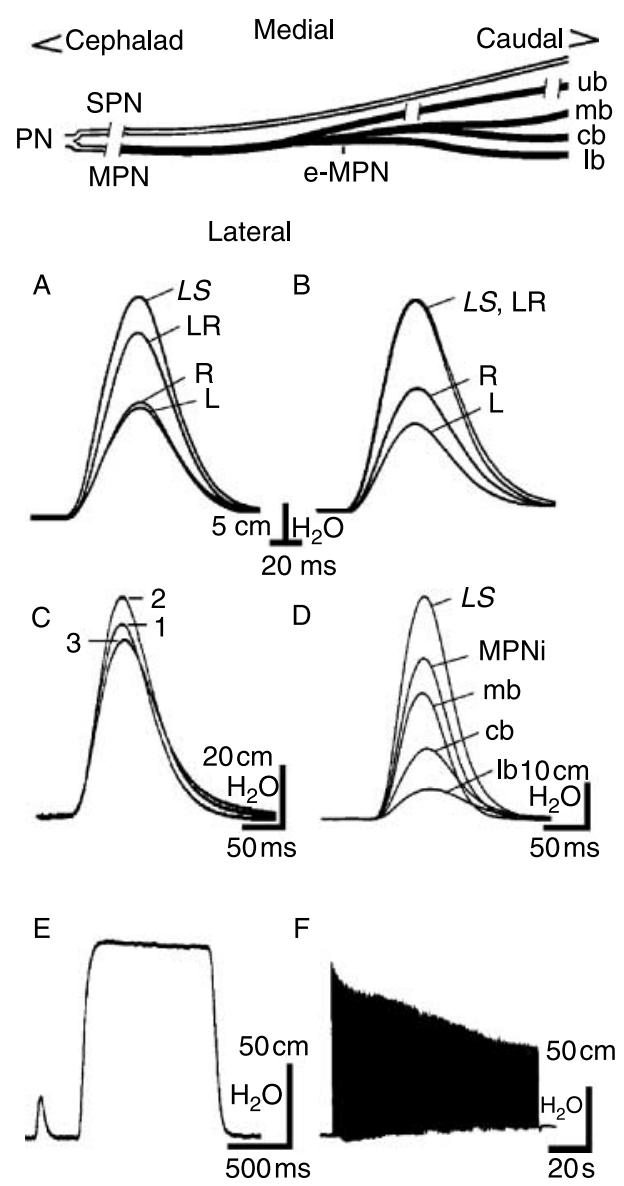

Figure 1 The inset (top) schematizes the nerves dissected in the ischial cavity of the left side (dorsal approach). PN, pudendal nerve trunk; SPN, sensory pudendal nerve; MPN, motor pudendal nerve; ub, urethral branch; e-MPN, external branch of MPN. Terminal branches of e-MPN were dissected: mb, medial branch; $c b$, central branch; lb, lateral branch. Panels A-D show automatic averages $(N=30)$ of pressure waves $(\mathrm{Pv})$ recorded in the lumen of the vaginal vestibule. In panels A and B Pvs were induced by single shock applied to e-MPN of either the left side (L), the right side (R) or to e-MPN of both sides (LR). $L S$ is the automatic point-to-point digital summation of Pvs elicited by the separate stimulation of $L$ and $R$; in panel $A, L S$ is greater than $L R$, but in panel $B L S$ and $L R$ are approximately equal. In panel C e-MPN-LR was stimulated with intact levator ani and ischiocavernosus muscles (1), and when these muscles were detached from the vagina (2); as shown, Pv is larger after the detachment of muscles. Pv was also induced by direct stimulation of the vestibule wall after the detachment of these muscles (3). The Pvs in panel D were induced by separate stimulation of the terminal e-MPN branches shown in the inset, or of the entire nerve; $\mathrm{mb}$ always induced the largest Pv. Panel $\mathrm{E}$ is a single trace showing $\mathrm{Pv}$ (single shock) and the pressure change stimulating at $40 \mathrm{~Hz}$ (tetanus). In panel F, repetitive tetanus at $40 \mathrm{~Hz}$ was applied to eMPN-L at one per second, each tetanus lasting $350 \mathrm{~ms}$.

related to body weight or vestibule length $(16-21 \mathrm{~mm})$. Pv-R was on average larger than Pv-L (Fig. 1B), but the difference was not statistically significant.

Time-to-peak and relaxation to half the peak amplitude of $\mathrm{Pv}-\mathrm{LR}$ were respectively $49.4 \pm 1.2 \mathrm{~ms}$ (S.E.M. of the averages) and $30.7 \pm 0.99 \mathrm{~ms}$. VCS reached complete tetanus ( $\mathrm{T}$ ) between 30 and $40 \mathrm{~Hz}$
(e-MPN-LR stimulation; Fig. 1E and F). The ratio of peak amplitudes $\mathrm{T} / \mathrm{Pv}$ was on average $4.5 \pm 0.6$. Using trains $350 \mathrm{~ms}$ long at $1 / \mathrm{s}, \mathrm{Pv}-\mathrm{LR}$ decayed to about half in $2 \mathrm{~min}$ (Fig. 1F). This is within the range of fatigability of motor units in the cat hind limb (Fig. 1F; Burke et al. 1971, 1973, 1974).

A bipolar stimulating electrode was fixed to the peripheral stump of e-MPN-L. Then, the cat $(N=3)$ was placed supine in order to search ventrally for the nerve and muscle that might be involved in vestibular constriction. After removal of superficial tissues, the ischium ramus and the obturator muscles, the distal vagina appeared to be covered ventrally and laterally by the halves of the levator ani muscle (LAM; Crouch \& Lackey 1969, Martin et al. 1974). LAM did not respond to e-MPN-L shock but the thin ischiocavernosus muscle (ICM; see Crouch \& Lackey 1969) and the vestibular wall did. After detaching and retracting LAM and ICM, the peak Pv-L was larger (compare Pvs 1 and 2 in Fig. 1C). Caudal to LAM, fragile venous sinuses and fat covered the ventral side of the vestibule. The fat was removed and a bipolar electrode was placed over the sinuses. Single shocks induced $\mathrm{Pv}$ and visible twitch of the vestibule wall (se Pv 3 in Fig. 1C). No other muscles were seen to twitch.

In each cat, the timing of unilateral and bilateral Pvs was comparable. The area under Pvs was automatically computed from digital recordings using a software program (Origin 5.0, Origin Lab Corporation, Northampton, MA, USA) that can add point-to-point the area of two or more Pvs ( $L S)$; Fig. $1 \mathrm{~A}$ and $\mathrm{B}$ show examples of $L S$. The $L S$ of unilateral Pvs was significantly larger than Pv-LR $(N=14 ; \quad P=0.025$, $t$-test for paired samples; Fig. 2) although in $3 / 14$ cats $L S$ and $P v-L R$ were approximately equal (difference $<0.5 \%$; Fig. $1 \mathrm{~B}$ ) and in one cat the latter was larger. The significant difference suggests that some VCS fibres might receive input from at least two motor axons, one from each side of the body.

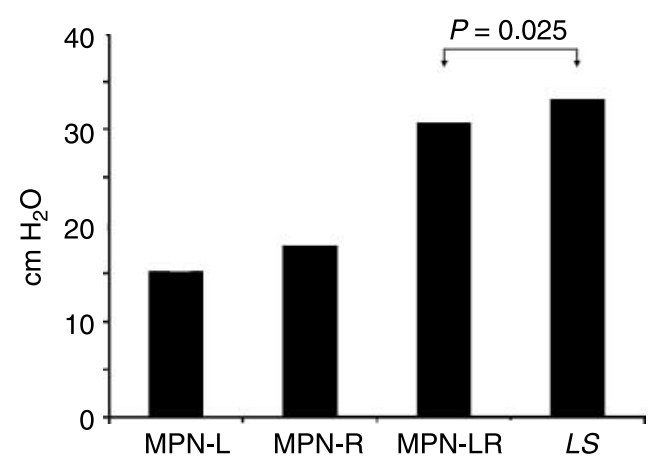

Figure 2 Averaged $\mathrm{Pv}$ peaks value $\left(\mathrm{cm} \mathrm{H}_{2} \mathrm{O}\right)$ from 14 cats. Thirty samples in each average. $L S$ is larger than $L R(P=0.025, t$-test for paired samples). Abbreviations as in Fig. 1. 


\section{The ICM does not increase the lumen pressure}

ICM received a small e-MPN branch (ventral approach) that induced an electromyogram (EMG) potential in ICM but no Pv (Fig. 3A). Stimulating another neighbour branch induced EMG potential in the vaginal wall as well as Pv, but no ICM potential (Fig. 3B). Thus, the ICM does not act as a vestibular constrictor. The muscle that produces Pv might be located below the venous sinuses.

\section{MPN branches inducing vestibule and orifice constriction}

Searching for a nerve branch that might innervate VCS only, we found (dorsal approach; $N=6$ ) that in most cats e-MPN divides into three branches (medial, central and lateral) joined together by connective tissue; separate stimulation of these branches induced Pvs with different amplitude $(N=6$; Fig. $1 \mathrm{~F}$ and Fig. 4$)$. $L S$ of the Pvs induced by separate branches stimulation was $27 \%$ greater than the $\mathrm{Pv}-\mathrm{LR}(P=0.02$; Fig. 2$)$. This suggests that some VCS fibres might receive more than one ipsilateral endplate.

The external orifice of the vagina (the introitus) and the anus as well constricted in response to nerve stimulation; the constrictions could not be measured. The medial e-MPN branch induced the largest Pv (Fig. 1D), but the introitus constriction was barely visible. The lateral branch induced opposite effects. This suggests that different muscles might produce $\mathrm{Pv}$ and introitus constriction independently.

Peak Pv-L is about twice in Fig. 4 than in Fig. $2(P=9.1$ E-6). The data in Fig. 2 were obtained during late autumn and winter, and those in Fig. 4 during spring-summer
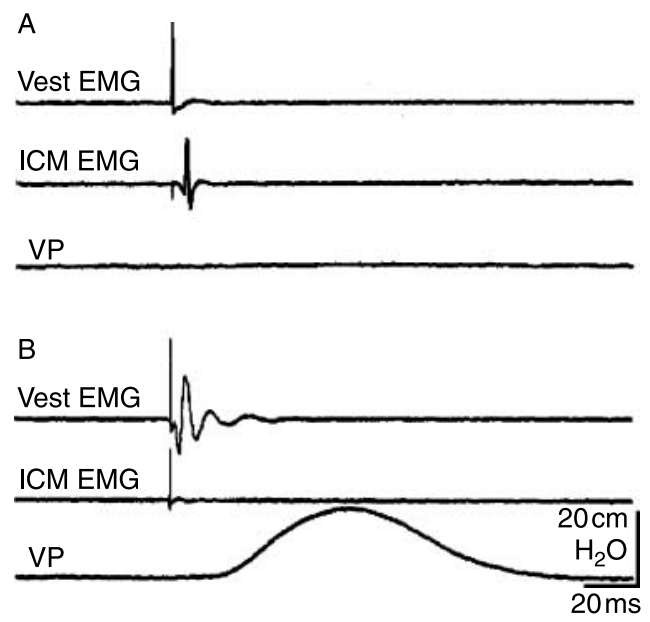

Figure 3 EMGs recorded with bipolar ball electrodes placed on the wall of the vaginal vestibule (Vest EMG) and on the EMG of the ischiocavernosus muscle (ICM) of the left side and lumen pressure of the vaginal vestibule (VP). In panel A, stimulation of an e-MPN-L branch induces neither Pv nor Vest EMG potential, but it did induce ICM EMG. In panel B, stimulation of another e-MPN-L branch induced both Vest EMG and Pv, but did not induce ICM EMG.
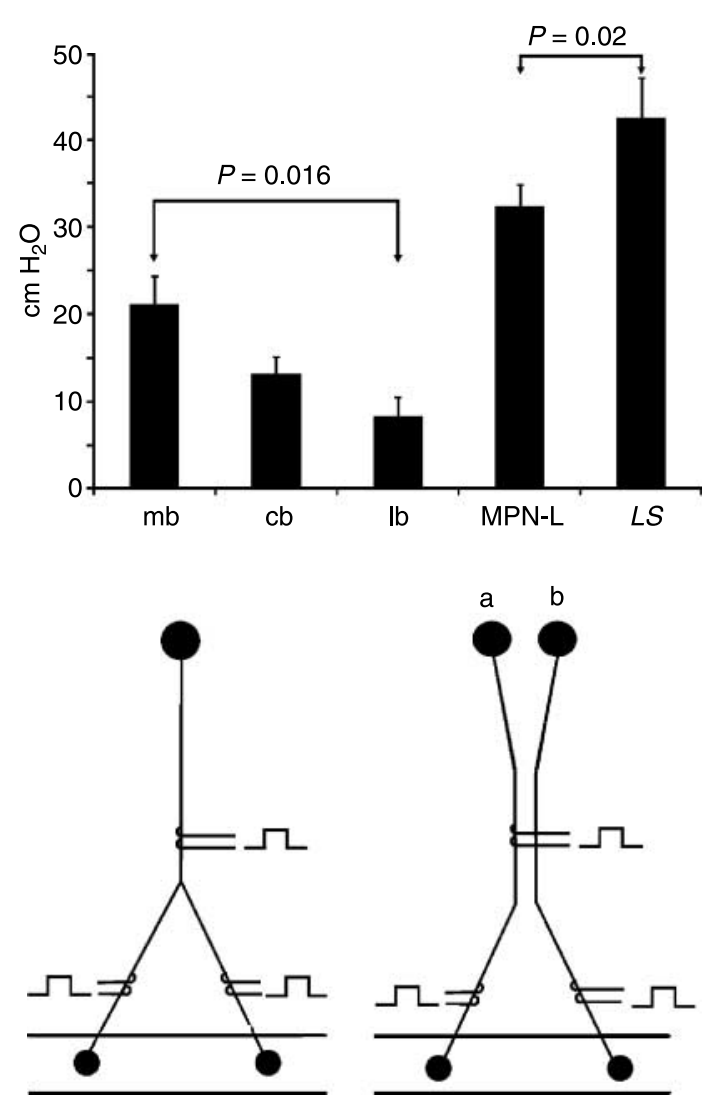

Figure 4 Peak value of average $\mathrm{Pv}(\mathrm{N}=6)$ induced by stimulation of the cat e-MPN-L or its branches. $\mathrm{Pv}$ induced by $\mathrm{mb}$ is significantly larger than the Pv induced by lb (paired $t$-test). $L S$ of the branches Pv is larger than the Pv induced by stimulation of the entire e-MPN-L ( $t$-test, paired samples). The diagrams at the bottom illustrate the two possibilities that might explain that the $L S$ of the branches $\mathrm{Pv}$ can be larger than the Pv induced by the stimulation of the entire nerve. In the diagram on the left, endplates from the same parent axon would be redundant, but endplates from different motoneurones ( $a$ and $b$; diagram on the right) might not. Abbreviations as in Figs 1 and 2).

and early autumn. The difference might be related to hormonal changes (see Komisaruk \& Adler 1972).

\section{Reflex e-MPN response to stimulation of the sensory pudendal nerve and to vestibule probing}

With the nerves intact, SPN-L was stimulated and the electroneurogram of e-MPN-L was recorded. Single shocks induced a reflex e-MPN-L discharge with latency of $8 \pm 1.24 \mathrm{~ms}(N=14$; Fig. $5 \mathrm{~A})$ as well as a Pv of about half the peak amplitude of the PV induced by e-MPN-L stimulation. In addition, the vestibule was probed $(N=8)$ at $4-10 \mathrm{~mm}$ from the introitus with probe displacement $<1 \mathrm{~mm}$. Single probing with 8-12 ms long displacement of the probe induced two e-MPN-L bursts (Fig. 5B). The latency of the first burst was $\cong 1 \mathrm{~ms}$ from the onset of probing; at the time of the burst onset, the displacement of the probe was $60-80 \mu \mathrm{m}$ (Fig. 5B). Given the short latency, this burst might originate in muscle afferents. 
A

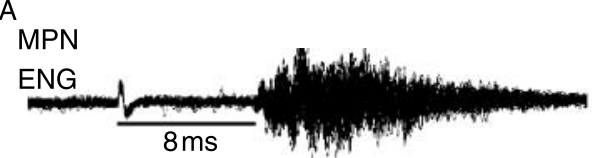

B

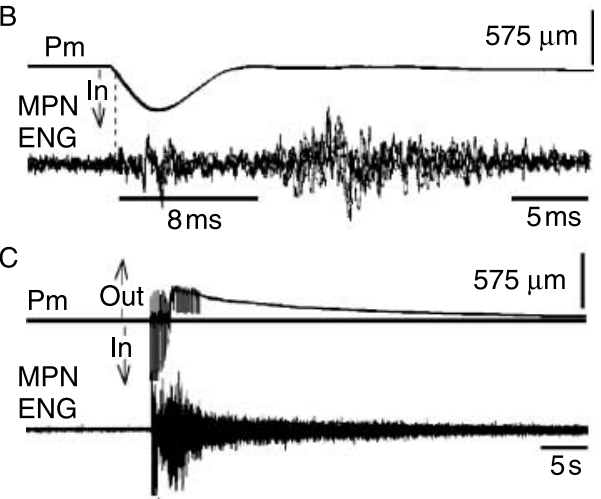

Figure 5 Reflex responses of MPN. In panel A, the sensory pudendal nerve (SPN) was stimulated; the latency of the reflex response is $\cong 8 \mathrm{~ms}$ (30 superimposed tracings). In panel $\mathrm{B}$, the vestibule was probed at $1 \mathrm{~cm}$ from the introitus; downward deflection indicates probe movement toward the vagina (Pm; middle tracings). The e-MPN-L discharge shows two bursts (eight superimposed tracings). The first burst started at $\mathrm{Pm} \cong 80 \mu \mathrm{m}$ (vertical interrupted line; latency $\cong 1 \mathrm{~ms}$ ). This short latency can be attributed only to activation of afferents in e-MPN-L. The second burst started $\cong 8 \mathrm{~ms}$ after the onset of the first burst. Panel C illustrates the effect of probing at $10 \mathrm{~Hz}$ during $5 \mathrm{~s}$ (single tracing). The e-MPN-L discharge is maximal during probing and was followed by prolonged after-discharge.

The delay of the second e-MPN-L burst is about the same as that of the reflex elicited by SPN-L shock.

Repetitive probing at $10 \mathrm{~Hz}$ during $5 \mathrm{~s}(\mathrm{~N}=4)$ induced a e-MPN-L after-discharge lasting $>1 \mathrm{~min}$ (Fig. $5 \mathrm{C}$ ) as it was induced in hindlimb muscles by vaginal probing (Cueva-Rolón et al. 1993) or SPN-L stimulation (Cueva-Rolón et al. 2002, Raya et al. 2004), and in pudendal efferent axons in response to noxious stimulation of the anal mucosa (Krier 1985). The probe movement (Pm in Fig. 5) showed two components (in-out movement), the inward movement being larger. The amplitude of the probe movement decreased progressively but mainly the inward movement (see arrows in Fig. 5), suggesting increasing vestibule resistance to probing. In addition, a slow outward movement of the probe appeared during stimulation and decreased steadily during the after-discharge. Thus, VCS contraction produces a tendency to drive out the probe and, presumably, the penis during mating.

The reflex induced by pudendal afferents in cats under barbiturate anaesthesia might suggest a high synaptic efficacy, resulting at least in part from reduced presynaptic inhibition (Weakly 1969, Nicoll 1975).

\section{Structural findings}

After fixation, the distal vagina was sectioned transversally in $5 \mathrm{~mm}$ blocks using scalpel $(N=3)$. The exposed faces of each block were photographed. The caudal face in Fig. $6 \mathrm{~A}$ is at $10 \mathrm{~mm}$ from the vaginal opening. At this location, the lumen is much wider than at $15 \mathrm{~mm}$. In blocks fixed with glutaraldehyde-osmium, a ring of dark tissue follows the transversal contour of the wall. The ring is made of striated muscle (Fig. 6C and D). This is the vestibule constrictor sphincter (VCS) that is situated between an internal layer of smooth muscle and connective tissue and an external adventitia and venous sinuses. VCS fibres ending freely were found at the dorsal and ventral midlines (Fig. 6E). To find this was a difficult task because most muscle fibres do not end exactly at the same level and optical plane. Figure $6 \mathrm{E}$ shows the end of four fibres in the dorsal midline. The fibre trajectory from one end to the other and does not follow the same plane as the plane of the transversal sections of VCS. Thus, we were unable to find both ends single fibres. Nonetheless, it is fair to say that VCS forms a ring of sphincter halves,
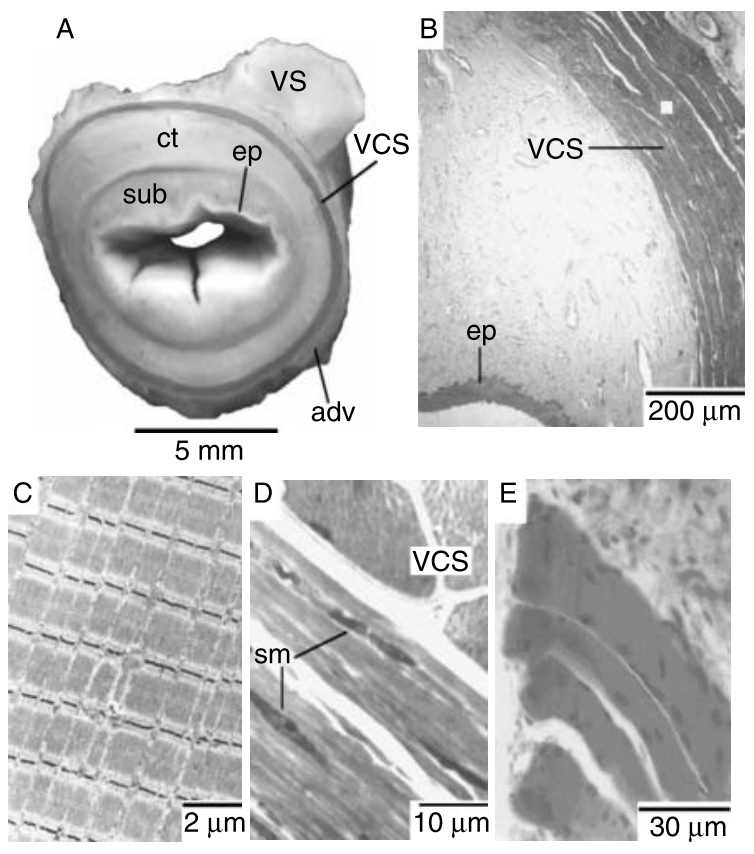

Figure 6 Structure and location of the vestibular constrictor sphincter (VCS) in the cat. A $5 \mathrm{~mm}$ thick block of the vestibule between 10 and $15 \mathrm{~mm}$ from the introitus (glutaraldehyde fixation) was taken. Panel A is photograph of the caudal, transversal face of the vestibule; ventral side is upward. The lumen is narrower at $15 \mathrm{~mm}$. VCS is the darker ring slightly retouched to increase contrast. The ring appears complete, but muscle fibres end freely at the dorsal and ventral middle lines (not shown); vs, venous sinus; adv, adventitia; ep, epithelium; sub, submucosa; $\mathrm{ct}$, connective tissue. Panel B is a transverse section $5 \mu \mathrm{m}$ thick, paraffin embedded, haematoxylin-eosin staining. Adventitia is in the upper right corner. A $1 \mathrm{~mm}^{3}$ piece was taken for EM from the area indicated in panel $B$ as a clear quadrangle. Panel $C$ is an EM image of VCS. Panel D shows a thin section (optic microscopy, epoxy embedding; toluidine blue staining) taken parallel to the main vestibule axis. Transversally sectioned fibres are seen at the upper right; below are longitudinal bundles of smooth muscle fibres; sm, smooth muscle. The photomicrograph in panel E shows the end of four VCS fibres in the dorsal midline. As explained in the text, to find these ends is a difficult task. 
which is in consonance with physiological results. (Fig. $1 \mathrm{~A}$ and $\mathrm{B}$ ). The ring extends $10-15 \mathrm{~mm}$ along the vestibule starting at $5-7 \mathrm{~mm}$ from the external orifice. Close to its cephalad and caudal limits, VCS separate ventrally then adopting a horseshoe shape (not shown). Longitudinal bundles of smooth muscle cells were localized close to deeper striated fibres (Fig. 6D).

\section{A correction to previous data}

The present data correct an earlier report. In a previous paper, slow adapting receptors were apparently activated in the cervix uteri (Cueva-Rolón et al. 1994). It was then thought that the cat cervix is at about $20 \mathrm{~mm}$ from the vaginal opening since a $4 \mathrm{~mm}$ wide probe could not be advanced any further. In present experiments, a $1 \mathrm{~mm}$ wide needle could be advanced $60 \mathrm{~mm}$, approximately, into the vaginal lumen with no obstacle. Histological analysis showed that the uteri localizes at this level (see Watson \& Glover 1993, Zambelli \& Cunto 2005). Thus, the recorded axons were not originated in the cervix but in the deeper part of the vestibule.

\section{Discussion}

\section{Targets of the MPN}

According to prior knowledge, the external branch of the MPN (e-MPN) only innervates the external anal sphincter (EAS) in the cat (Thor et al. 1989, Fedirchuk et al. 1992, Paroschy \& Shefchyk 2000). VCS and ICM can be added to the list. Furthermore, e-MPN might also innervate an introitus constrictor muscle since some of its branches induce clear visible orifice constriction but small increase in vestibule pressure and other branches induce the opposite effect. Constriction of the vaginal introitus might be produced by the vulvae constrictor described by Martin et al. (1974) in post-mortem, fixed cats.

\section{Different vestibuli constrictor muscles?}

There is little doubt that the VCS studied here increases the vestibular pressure. The timing of the twitch pressure $(\mathrm{Pv})$ is close to the twitch tension wave of hind limb muscle units (Burke et al. 1971, 1973, 1974, Lewis 1972) and of the male bulbospongiosus (bulbocavernosus) muscle in vitro (Bowen et al. 1984). The tetanus/twitch tension ratio was close to that of the MG muscle (Lewis 1972).

Three different muscles had been named vestibuli constrictor, two in the cat (Crouch \& Lackey 1969, Martin et al. 1974) and one in the rabbit (MartínezGómez et al. 1997, Cruz et al. 2002). The descriptions were based on fixed cadavers. These muscles do not resemble VCS. The vestibular constrictor described by Crouch \& Lackey (1969) will be here VC1; it arises in the EAS, surrounds the vestibule up to the external orifice and inserts in the urogenital sinus. The VCS neither reaches the orifice nor does it have this insertion.

Martin et al. (1974) also stated that fascicles of EAS form a vestibuli constrictor (VC2) that is situated on the lateral wall of the vestibule but does not encircle it; the majority of VC2 fibres insert ventrally in the ischial arc. In contrast to the VC2, the VCS entirely surrounds the vestibule and the muscle fibres do not show bone insertion but end freely in the vestibule wall. Thus, neither the VC1 nor the VC2 correspond to the VCS.

The vestibuli constrictor (VC3) described in the rabbit (Martínez-Gómez et al. 1997, Cruz et al. 2002) has a bone insertion. Vaginal pressure increased by separate contraction of ICM and the bulbospongiosus muscle (BSM; Cruz et al. 2002). In the cat, the ICM does not produce $\mathrm{Pv}$ and the BSM was not identified in the female either by us or by Crouch \& Lackey (1969) or Martin et al. (1974). Differences between species should be considered, but a sphincter similar to VCS might be present in other species. We do not believe that the cat VCS is an exception among mammals, including humans. Women's orgasm initiates with involuntary, rhythmic contractions of circumvaginal striated muscles (for review, Weston et al. 2004), and similar contractions are induced by stimulation of the clitoris (Shafik et al. 2008). We wonder whether a sphincter similar to VCS might be involved in these responses. We wonder also whether the VCS might correspond to the BSM in the male.

The magnitude of $\mathrm{Pv}$ or $\mathrm{T}$ cannot be compared with previous data from the rat (Pacheco et al. 1989) and the rabbit (Cruz et al. 2002); these authors give the lumen pressure in force units (grams), but there is no way to convert grams to $\mathrm{cm}$ of water.

\section{Multiple innervation of VCS}

Dogma states that a single muscle fibre receive a single motor terminal. Now, there is enough evidence to reject this dogma. (Sandmann 1885, quoted by Katz \& Kuffler 1941, Jarcho et al. 1952, Rossi 1990, Zenker et al. 1990, Duxson \& Sheard 1995, Happak et al. 1997, Perie et al. 1997, Lateva et al. 2002). Present results might also challenge the dogma. The sum of the unilaterally induced Pvs was larger than the Pv induced by e-MPN$L R$, suggesting that some single muscle fibres might be innervated by e-MPN axons from both sides. We wonder whether this might occur in other medial muscles.

In addition, the sum of Pvs induced by maximal stimulation of e-MPN branches was larger than the Pv induced by maximal stimulation of the entire MPN. From these data, it can be inferred that some single VCS fibres might receive more than one terminal, each one inducing threshold end-plate potential (e.p.p.). In normal conditions, two or more endplates would be redundant if supplied by a single e-MPN axon that divides into daughter fibres travelling in different nerve 
branches. Thus, stimulating the parent e-MPN axon or each branch separately would produce muscle fibre firing (Fig. 4, diagram on the left). Alternatively, different motoneurones might innervate a single muscle fibre (Fig. 4, diagram on the right). Let us assume that a single muscle fibre receives motor terminals from both motoneurones, $a$ and $b$. At a given moment, neuron $a$ might fire but not neuron $b$. In another moment, neuron $b$ might fire but neuron a does not, but at both moments the muscle fibre contracts. This would increase certainty in the motor system in spite of uncertainty in the central transmission.

\section{Materials and Methods}

\section{General procedure}

Thirty-five adult female cats weighing $2.5-3.6 \mathrm{~kg}$ were used. The CINVESTAV Internal Committee for Care of Laboratory Animals approved the protocols based on the observance of the Mexican Norm for the Use of Laboratory Animals (NOM-062ZOO-1999). The cats were anaesthetized by i.p. injection of pentobarbitone $(35 \mathrm{mg} / \mathrm{kg}$; Pfizer, México D. F.) plus additional i.v. doses as needed. The cats did not react to noxious stimulation (pupil diameter, heart frequency and withdraw reflex).

The iliac bone was fixed bilaterally with the cat in prone decubitus (see Cueva-Rolón et al. 1993). The trunk and branches of the pudendal was exposed in the ischial fossa on both sides of the body. In all experiments, the right pudendal nerve was sectioned close to the sciatic notch. The neighbour posterior femoral nerve (Fig. 4 in Langley \& Anderson 1896), which is a cutaneous nerve, was also sectioned at the same level. The ischial fossa was covered with mineral oil that was maintained at around $37^{\circ} \mathrm{C}$ by radiant heat. The left pudendal trunk was either sectioned or kept intact. The pudendal trunk divides into a sensory branch (SPN) and a motor branch (MPN). MPN divides into the internal or urethral and external branches (e-MPN), which was exposed for electrical stimulation or, in some experiments, for recording. In the present experiments, the urethral branch was sectioned distally. SPN and e-MPN were mounted on bipolar Ag electrodes for stimulation or recording.

The bulb of a number 12 Foley catheter that was tightly plugged to a pressure transducer. The bulb of the catheter was introduced as far as possible $(16-21 \mathrm{~mm})$ in the lumen of the vaginal vestibule (see Crouch \& Lackey 1969, Watson \& Glover 1993, Zambelli \& Cunto 2005). The bulb (25 mm long, spindle shaped and $4 \mathrm{~mm}$ wide in its wider, central portion) could not be advanced further due to progressive narrowing of the vestibular lumen in the cephalad direction. The length of the vestibule is close to the length of the erect penis (Watson \& Glover 1993).

\section{Probing the vaginal vestibule}

The vestibule was probed with a probe displacement of less than $1 \mathrm{~mm}$ (Cueva-Rolón et al. 1993). The probe was an aluminium cylinder with a probing tip $8 \mathrm{~mm}$ long and $6 \mathrm{~mm}$ in diameter covered by rubber. The entire probe was placed inside a slightly wider, hollow cylinder (the carrier) that was shorter than the probe. Thus, the probe ends emerged at both sides of the carrier, which was attached to a manipulator. A spring joining the carrier and the probe was stretched when the latter moved forward by tapping on its rear end, and recoiled after tapping. The rear end of the probe was attached to one end of the cantilever pole of a modified version of Statham strain gauge (Delgado-Lezama et al. 1997). A mirror attached perpendicularly to the mobile cantilever pole reflects the light supplied by an optocoupler (OC) fixed to the strain gauge and connected to a constant current amplifier. The $\mathrm{OC}$ sends a light beam to the mirror, and converts linearly the reflected light to output voltage; reflected light depends linearly on the distance between the mirror and the OC beam. The OC output was connected to the recording instrument. The probe tip was introduced in the vestibule using the manipulator holding (the carrier). A $4 \mathrm{~mm}$ wide pole of a plastic material was attached to the movable centre of a modified load speaker (electromagnetic coil; EMC), which was held to a manipulator and fed with a conveniently amplified square pulse. The free end of the EMC pole was concave, and it was positioned in close contact with the complementary convexity of the transducer free end pole, which moves the strain guage contilever. Therefore, any movement of the EMC pole was transmitted to both the transducer pole and the vaginal probe and vice versa. The probe movement was close to a half-sinusoidal wave. Thus, the probing device consists of the in-series arrangement of probe, the modified strain gauge and the EMC. The strain gauge output was used to monitor the probe movement. Additional details are given in the results.

At the end of the experiments, the cats were killed by an overdose of pentobarbitone.

\section{Statistical analysis}

Automatic average $(N=30-100)$ of the pressure wave $(\mathrm{Pv})$ induced by single electric shocks was used to estimate the peak pressure, the time to peak, decay time to half the peak pressure an area under the pressure wave using a software program (Origin 5.0). The S.E.M. of the average was used to estimate the variability of these parameters; the S.D. of these parameters in the samples of each average was less than $5 \%$. The $t$-test was used to find statistical significance between paired averages.

\section{VCS structure}

Just after killing the cat, the vestibule with part of the distal vagina was excised and fixed either with $1.25 \%$ glutaraldehyde in $0.1 \mathrm{M}$ cacodylate solution for electron microscopy (EM) or light microscopy, or with buffered $4 \%$ paraformaldehyde for light microscopy. The fixed vestibule was transversally divided with scalpel into $5 \mathrm{~mm}$ thick blocks that were photographed. Transverse slices $5 \mu \mathrm{m}$ thick embedded in paraffin were stained with haematoxylin-eosin for light microscopy. Specimens of about $1 \mathrm{~mm}^{3}$ post-fixed with osmium were processed for epoxy embedding. Transversal or longitudinal semi-thin sections were used for light microscopy staining with toluidine blue or with uranyl acetate and lead citrate for EM. Additional details are given in the section of results. 


\section{Declaration of interest}

The authors declare that there is no conflict of interest that could be perceived as prejudicing the impartiality of the research reported.

\section{Funding}

This research did not receive any specific grant from any funding agency in the public, commercial or not-for-profit sector.

\section{Acknowledgements}

The content of this paper is part of the thesis of R. LagunesCórdoba to obtain the PhD at the Departamento de Fisiología, Biofísica y Neurociencias, CINVESTAV. The authors are indebted to José Guadalupe Raya and Mario Raya for technical assistance and to QFB Silvia Galindo Gómez and MSc Angélica Silva-Olivares for the preparation of morphological material.

\section{References}

Bowen JM, Hughes BJ \& Bradley WE 1984 Anatomic, physiologic and pharmacological properties of the feline bulbospongiosus muscle. American Journal of Veterinary Research 45 308-313.

Burke RE, Levine DN, Zajac FE, Tsairis P \& Engel WK 1971 Mammalian motor units: physiological-histochemical correlation in three types in cat gastrocnemius. Science 174 709-712.

Burke RE, Levine DN, Tsairis P \& Zajac FE 1973 Physiological types and histochemical profiles in motor units of the cat gastrocnemius. Journal of Physiology 234 723-748.

Burke RE, Levine DN, Salcman M \& Tsairis P 1974 Motor units in cat soleus muscle: physiological, histochemical and morphological characteristics. Journal of Physiology 238 503-514.

Crouch JE \& Lackey MB 1969 Text-Atlas of Cat Anatomy, Philadelphia, USA: Lea and Febiger.

Cruz Y, Hudson R, Pacheco P, Lucio RA \& Martínez-Gómez M 2002 Anatomical and physiological characteristics of perineal muscles in the female rabbit. Physiology and Behavior 75 33-40.

Cueva-Rolón R, Muñoz-Martínez EJ, Delgado-Lezama R, Raya JG \& González-Santos G 1993 Sustained activation of the triceps surae muscles produced by mechanical stimulation of the genital tract of the female cat. Brain Research 600 33-38.

Cueva-Rolón R, Muñoz-Martínez EJ, Delgado-Lezama R \& Raya JG 1994 The cat pudendal nerve: afferent fibres responding to mechanical stimulation of the perineal skin, the vagina or the uterine cervix. Brain Research 655 1-6.

Cueva-Rolón R, Delgado-Lezama R, Raya JG, Raya M, Tecuanhuey R \& Muñoz-Martínez EJ 2002 Sustained firing of alpha and gamma hind limb motoneurones induced by stimulation of the pudendal nerve. Journal of Neurophysiology 88 3232-3242.

Delgado-Lezama R, Raya JG \& Muñoz-Martínez EJ 1997 Methods to find aponeurosis and tendon stiffness and the onset of muscle contraction. Journal of Neuroscience Methods 78 125-132.

Duxson MJ \& Sheard PW 1995 Formation of new myotubes occurs exclusively at the multiple innervation zones of an embryonic large muscle. Developmental Dynamics 204 391-405.

Fedirchuk B, Hochman S \& Shefchyk SJ 1992 An intracellular study of perineal and hindlimb afferent inputs onto sphincter motoneurones in the decerebrate cat. Experimental Brain Research 89 511-516.

Happak W, Liu J, Burggasser G, Flowers A, Gruber H \& Freilinger G 1997 Human facial muscles: dimensions, motor endplate distribution, and presence of muscle fibres with multiple motor endplates. Anatomical Record 249 276-284.

Jarcho W, Eyzaguirre C, Berman B \& Lilienthal JL 1952 Spread of excitation in skeletal muscle: some factors contributing to the form of the electromyogram. Journal of Physiology 168 446-457.

Katz B \& Kuffler SW 1941 Multiple motor innervation of the frog's sartorius muscle. Journal of Neurophysiology 4 209-223.
Komisaruk BR \& Adler NT 1972 Genital sensory field: enlargement by estrogen treatment in female rats. Science 178 1295-1298.

Krier J 1985 Discharge patterns of pudendal efferent fibres innervating the external anal sphincter of the cat. Journal of Physiology 366 471-480.

Langley JN \& Anderson HK 1896 The innervation of the pelvic and adjoining viscera. Part III. Journal of Physiology 19 85-121.

Lateva ZC, McGill KC \& Johanson ME 2002 Electrophysiological evidence of adult human skeletal muscle fibres with multiple endplates and polyneuronal innervation. Journal of Physiology 44 549-565.

Lewis DM 1972 The effect of denervation on the mechanical and electrical responses of fast and slow mammalian twitch muscle. Journal of Physiology 222 51-75.

Martin WD, Fletcher TF \& Bradley WE 1974 Perineal musculature in the cat. Anatomical Record 180 3-14.

Martínez-Gómez M, Lucio RA, Carro M, Pacheco P \& Hudson R 1997 Striated muscles and scent glands associated with the vaginal tract of the rabbit. Anatomical Record 247 486-495.

Nicoll RA 1975 Presynaptic action of barbiturates in the frog spinal cord. PNAS 72 1460-1463.

Oelrich TM 1983 The striated urogenital sphincter muscle in the female. Anatomical Record 205 223-232.

Pacheco P, Martínez-Gómez M, Whipple B, Beyer C \& Komisaruk BR 1989 Somato-motor components of the pelvic and pudendal nerves of the female rat. Brain Research 490 85-95.

Paroschy KL \& Shefchyk SJ 2000 Non-linear membrane properties of sacral sphincter motoneurones in the decerebrate cat. Journal of Physiology 523 741-753.

Perie S, St Guily JL, Callard P \& Sebille A 1997 Innervation of adult human laryngeal muscle fibres. Journal of Neurological Sciences 149 81-86.

Raya JG, Ramírez A \& Muñoz-Martínez EJ 2004 Gamma $\rightarrow$ alpha linkage and persistent firing of la fibres by pudendal nerve stimulation in the decerebrate cat. Journal of Neurophysiology 92 387-394.

Rossi G 1990 From the pattern of human vocal muscle fibre innervation to functional remarks. Acta Oto-Laryngologica 473 1-10.

Sandmann G 1885 Ueber die Vertheilung der motorischen Nervenendapparate in den quergestreiften Musklen der Wirbelthiere. Quoted in: Lateva ZC, McGill KC, Johanson ME 2002 Electrophysiological evidence of adult human skeletal muscle fibres with multiple endplates and polyneuronal innervation. Journal of Physiology 544 549-565.

Shafik A 1993 Vaginocavernosus reflex. Clinical significance and role in sexual act. Gynecologic and Obstetric Investigation 35 114-117.

Shafik A, El Sibai O, Shafik AA, Ahmed I \& Mostafa RM 2004 The elctrovaginogram: study of the vaginal electric activity and its role in sexual act and disorders. Archives of Gynecology and Obstetrics 269 282-286.

Shafik A, El Sibai O \& Shafik AA 2008 Vaginal response to clitoral stimulation: identification of the clitorovaginal reflex. Journal of Reproductive Medicine 53 111-116.

Thor KB, Morgan C, Nadelhaft I, Houston M \& De Groat WC 1989 Organization of afferent and efferent pathways in the pudendal nerve of the female cat. Journal of Comparative Neurology 288 263-279.

Watson PF \& Glover TE 1993 Vaginal anatomy of the domestic cat Felis catus in relation to copulation and artificial insemination. Journal of Reproduction and Fertility 47 355-359.

Weakly JN 1969 Effect of barbiturates on 'quantal' synaptic transmission in spinal motoneurones. Journal of Physiology 204 63-77.

Weston CM, Levin RJ, Sipski ML, Hull EM \& Heiman JR 2004 Women's orgasm. Annual Review of Sex Research 15 173-257.

Zambelli D \& Cunto M 2005 Vaginal and cervical modifications during the estrus cycle in the domestic cat. Theriogenology 64 679-684.

Zenker W, Snobl D \& Boetschi R 1990 Multifocal innervation and muscle length. A morphological study on the role of myo-myonal junctions, fibre branching and multiple innervation in muscles of different size and shape. Anatomy and Embryology 182 273-283.

Received 4 August 2008

First decision 18 September 2008

Revised manuscript received 29 October 2008

Accepted 21 November 2008 